\title{
Hallmarks of Non-accidental Trauma: A Surgeon's Perspective
}

\author{
Nilda M. Garcia $\cdot$ Karla A. Lawson
}

Published online: 29 July 2014

(C) Springer Science + Business Media New York 2014

\begin{abstract}
Child abuse is a public health epidemic in the United States with high incidence, prevalence, and severe personal and societal impact. Surgeons should be considering child abuse in their treatment of children with traumatic injury. Screening for child abuse mechanism in the ED and in-patient settings is not precise, subject to bias and often incomplete. A variety of tools and factors have been studied for their predictive potential despite inherent methodological issues; thus, no one screening tool has been proposed as the standard of care. More research is warranted to better define how to best screen patients for the need for further child abuse investigation. Given the accumulative impact of ionizing radiation with children, the utilization of unnecessary CTs should be limited. For instance, the use of head CT in children suspected of inflicted head trauma should be limited; however, very few additional screening modalities exist to better identify the subset of children that need a CT. Studies on serum CSF biomarkers of head injury are promising and yet require further research in order to recommend their use in the clinical setting.
\end{abstract}

This article is part of the Topical Collection on Pediatric Trauma Surgery.

\author{
N. M. Garcia $(\bowtie) \cdot$ K. A. Lawson \\ Trauma Services Department, Dell Children's Medical Center of \\ Central Texas, 4900 Mueller Blvd., Austin, TX 78723, USA \\ e-mail: NMGarcia@seton.org \\ K. A. Lawson \\ e-mail: kalawson@seton.org
}

N. M. Garcia · K. A. Lawson

Department of Surgery, University of Texas Southwestern Medical School-Austin, 4900 Mueller Blvd., Austin, TX 78723, USA
Keywords Non-accidental trauma $\cdot$ Surgeon $\cdot$ Child abuse $\cdot$ Biomarkers $\cdot$ Screening $\cdot$ Constellation of symptoms · Differential diagnosis $\cdot$ Screening $\cdot$ Detection $\cdot$ Skeletal survey $\cdot$ Abdominal trauma $\cdot$ Head trauma

\section{Introduction}

The maltreatment of children is a significant public health concern in the United States and globally. The World Health Organization (WHO) defines maltreatment as "all forms of physical and/or emotional ill-treatment, sexual abuse, neglect and exploitation, resulting in actual or potential harm to a child's health, survival, development or dignity" [1]. Over 678,000 children were reported victims of maltreatment in the United States in 2012 [2]. Of these, more than $18 \%$ were victims of physical abuse leading to injury. The yearly incidence of child physical abuse in the United States is approximately 1:500 children. Over 1,600 children died from child maltreatment in 2012. Approximately half of all maltreatment deaths in the United States were attributed to physical abuse.

The incidence and prevalence of abuse may be high, but possibly more important are the cumulative detrimental effects of child maltreatment on the child, family, and society. Data from the Adverse Childhood Experience (ACE) Study, one of the largest studies to specifically investigate the later effects of child maltreatment on health, illuminate this public health concern [3]. A large body of scientific literature has emerged utilizing the data collected in the ACE Study. In the original study, almost 10,000 adults answered questions about adverse childhood experiences, and these experiences were then correlated with poor health outcomes [3]. There was a significant relationship between the number of adverse events suffered as a child and their adult health behaviors and disease. 
Alcoholism, drug abuse, and mental illness were 4-12 times more likely in those experiencing 4 or more types of adverse events in their childhood. Similar findings were true for smoking and sexual-related health factors. A smaller, but significant relationship was also found between adverse events and both lack of physical activity and obesity in this population. In addition to its relationship to risk factors, these adverse events were also related to the presence of adult-onset chronic diseases such as heart disease, cancer, chronic lung disease, skeletal fractures, and liver disease [3]. Recent and exciting scientific work is seeking to establish a causal connection between child maltreatment and health and well-being later in life. This work is focusing on the effect of adverse events on epigenetic changes and subsequent changes in gene expression.

Physician involvement in child abuse investigation and management is a relatively modern concept as many physicians believed their role was in the healing of physical injury, but not in the healing of societal issues such as child abuse [4]. In the 1940s, John Caffey published his work detailing six cases of unexplained child injury and later his work on "The Whiplash Shaken Infant Syndrome," prompted a string of published work on the topic and an eventual convening of a scientific conference and the publishing of the 1962 groundbreaking article by Dr. C. Henry Kempe, "The Battered Child Syndrome." This was the first nationally recognized article, which led to the awakening of physicians throughout the country to the problem of abused children in their care. By 1968, all fifty states had enacted mandatory reporting laws. Although pediatricians have since been keen advocates in the screening, diagnosis, and management of the battered child, surgeons have only recently begun to play a major role in this process. In 2013, Larimer et al. [5•] published the need for surgeon involvement in the evaluation of child abuse or non-accidental trauma and point to the need for surgeons to consider child abuse in their differential. This is particularly true, as catching child abuse in its earliest stages is paramount to preventing mortality. The literature shows that missed injury and multiple-incident child abuse are related to increase in mortality [6, 7]. In one study, those children with multiple diagnosed episodes of child abuse had significantly higher mortality than those diagnosed only a single time (24.5 versus $9.9 \%$ mortality, $p=0.002$ ) [6].

\section{Constellation of Symptoms/Differential Diagnosis}

When dealing with trauma, surgeons should consider child abuse in their clinical differential as well as play a key role in the clinical care and management of abused patients.
Missed diagnosis in child abuse is common and can be devastating. The involvement of the surgeon is key as much of abusive injury is poly-traumatic in nature.

Recent work by Larimer et al. [5•], points to the importance of surgeon involvement in the evaluation of non-accidental trauma. In their study of 267 victims of non-accidental trauma, $36 \%$ of victims had three or more separate areas of injury, with more than $40 \%$ requiring an interventional procedure or operation. When compared to other trauma patients, this population had a higher rate of ICU admission, length of stay, and increased mortality. Based on the poly-traumatic nature of child abuse, common need for surgical intervention, and increased severity of injuries for these patients, it becomes clear why surgeon involvement is necessary in the evaluation of these patients.

Physical abuse is difficult to detect and diagnose. Missed detection is not only common but can also be clinically devastating to the child. Seminal work of Dr. Carol Jenny and colleagues detailed the information on missed cases of abusive head trauma [7]. Thirty-one percent of a cohort of children diagnosed with abusive head trauma were evaluated in the emergency room following their head injury and failed to receive proper identification of true mechanism. The mean time to final diagnosis and detection of abuse for these children was 7 days. Almost half of these children experienced medical complications related to their missed diagnosis. These physicians concluded that 4 of the 5 deaths reported in this cohort could have been prevented by proper detection and subsequent treatment. This study highlights the large number of children we may be missing in terms of child abuse detection leading to increased injury and mortality for these children [6].

This phenomenon is not limited to children suffering abusive head trauma. In a cohort of children diagnosed with abusive fractures, more than $20 \%$ had a previous physician interaction and subsequent missed diagnosis [8]. The median time from first physician visit to eventual identification of abuse was 8 days. Based on this data, understanding the constellation of key symptoms and risk factors in the abused child is a requirement to good clinical care. Development of effective screening and detection guidelines are key to this endeavor.

\section{Detection of Child Abuse}

The physical injuries associated with abusive trauma disproportionally affect the most vulnerable populations, including the young, those with special needs, the premature and medically complex. Specifically, children less than one year of age have double the documented incidence of child maltreatment when compared to older children (20.6 per 1,000 children versus $<12$ per 1,000 in every 
other age group) [2]. As this population is developmentally unable to protect themselves via out-cry, it often falls to the clinical expert to detect and diagnose maltreatment. The un-biased detection and diagnosis of physical abuse are exceedingly difficult as clinicians have limited predictive measures and formal screening tools at their disposal, especially when a child presents with no definitive sign of physical trauma and no report of apparent life-threatening event. Because many decisions are based on intuition, clinicians demonstrate inherent bias in their screening decisions based on race and socio-economic factors of families. Emergency physicians are more likely to perform skeletal survey $(65.3$ versus $31.1 \%, p<0.001)$ and report (52.9 versus $22.5 \%, p<0.001)$ minority children to Child Protective Services (CPS) compared to their white counterparts [9]. This relationship remained significant after controlling for likelihood of inflicted injury.

Child abuse is difficult to detect, bias has been identified in the determination of child abuse in the emergency department, and missed child abuse leads to poor outcomes for children. Because of this, screening mechanisms to prevent bias and accurately identify abused children are needed. A number of studies report on the results of screening tools for use in the ED and inpatient settings. A recent systematic review identified and evaluated 7 manuscripts published on the screening and detection of physical child abuse for which diagnostic accuracy of the screen was available [10]. The authors deemed all studies identified to have some methodological or quality issues as defined by a priori criteria. Four studies were specific to head injury, while three evaluated general abusive injuries [11-17]. The studies looked at a variety of predictive factors for child abuse including individual criteria such as severe retinal hemorrhage, brain ischemia, presence of subdural hematoma, lack of history or low-impact trauma history, imaging patterns, bruise location consistent with fracture site, as well as tools integrating multiple factors such as a combination of bruise region, age of child, and mechanism history or a combination of age of child, physical exam findings, and imaging results. Of these studied factors and tools, only the individual variable, absence of scalp swelling [15], and the decision tool integrating bruising location, child age, and history [13], produced a sensitivity of greater than $90 \%$. Although findings for these 2 parameters/tools seem promising, the review authors point out methodological issues in all studies examined.

\section{Screening for Child Abuse}

Since the publication of this systematic review, several additional papers have reported on the investigation of clinical prediction rules or factors. Louwers et al. [18•] studied a prediction tool utilized in three Dutch emergency rooms. The screener included six items: Is the history consistent?, Was there a delay in presentation?, Is the injury consistent with developmental milestones?, Is the behavior of the child and caregivers appropriate?, and did the physical exam and history have signals that make you doubt the safety of the caregivers? Screening was completed on more than 18,000 children, aged $0-18$ years, presenting to an ED. A child abuse mechanism was defined via a child abuse investigation team at each hospital yielding 420 that screened positive. An independent review panel then re-evaluated forty-four children of the 420 that screened positive using the tool and 11 of 17,855 who did not. Sensitivity of the tool was only $80 \%$, while specificity was $98 \%$. These findings indicate that the tool had only a moderate ability to detect child abuse, though when the tool was negative, patients were unlikely to be abused.

Hymel et al. [19] sought to create a clinical prediction rule for abusive head trauma (AHT) in the pediatric intensive care setting. They collected variables available to most clinical staff early in case presentation that may be predictive of eventual child abuse determination. Based on their data, a 5-variable tool emerged showing $95 \%$ sensitivity and $95 \%$ specificity for abuse. The factors included in the tool were acute respiratory compromise, seizure or acute encephalopathy prior to admission, bruising to the ear, neck or torso, interhemispheric or bilateral subdural collection, and any complicated skull fracture. The authors caution clinicians to not use the prediction rule for a positive determination of AHT, but instead to help rule out AHT in those patients scoring negatively with the tool [19]. Critics of this tool cite the fact that $45 \%$ of the children in this study met definitional criteria for child abuse and with such a high pre-test probability of abuse, that everyone should be screened in this paradigm so a prediction rule on those that require screening is ill suited to rule out the need for screening [20]. Despite not promoting any particular screening modalities or predictive tools, a recent article presents findings on the implementation of a child abuse clinical guideline and its effect on bias in screening. The study showed that implementation of a guideline to screen all patients less than a year of age presenting to an ED with any type of fracture decreased bias in screening based on socio-economic status of the patient [21]. The study did show an increase in skeletal survey utilization, but did not increase CT utilization for these patients. One limitation of this study was its inability to investigate differences in child-base-related fractures specifically.

Use of Skeletal Survey in Screening

Skeletal injuries in young children can be red flags for inflicted injury. Fractures generally associated with child 
Table 1 Systematic review results for association of fracture location with abuse

\begin{tabular}{ll}
\hline Fracture location & Pooled estimate of probability \\
\hline Rib fracture & $0.71(0.42-0.91)$ \\
Femoral fracture & $0.43(0.32-0.54)$ \\
Humeral fracture & $0.54(0.20-0.88)$ \\
Skull fracture & $0.30(0.19-0.46)$ \\
\hline
\end{tabular}

See especially Kemp et al. [22]

abuse mechanism include rib fractures, certain orientation of long bone fractures including spiral and oblique fractures that may denote a twisting mechanism of injury, and metaphyseal fractures. A meta-analytic review of the literature by Kemp et al. [22] synthesized the available data on skeletal injury and child abuse mechanism finding a high probability for rib fracture and inflicted injury (Table 1). In addition, metaphyseal fractures of the long bones were more often identified in abused children compared to children suffering fractures via accidental mechanisms, though data was too sparse to conduct metaanalytic evaluation.

Skeletal survey is often utilized in screening for child abuse in the very young with the intent of finding unknown or unsuspected fractures, secondary to an American Academy of Pediatrics (AAP) directive to do so in all children less than 2 years of age suspected of being abuse victims [23]. A study looking retrospectively at the rate of newly identified fractures was conducted in 703 children over a four-year period receiving skeletal survey in their child abuse screening process [24•]. More than 1 out of every 10 patients receiving a skeletal survey in their child abuse investigation found new, un-suspected fractures. The majority of fractures found with skeletal survey were healing. Children who were less than 6 months of age were more likely to have positive skeletal survey results when compared to their older counterparts (16.4 versus $6.8 \%$, $p<0.001)$. In addition, presenting with an Apparent Life Threatening Event (ALTE) (18.2\% of ALTE patients, $p=0.05)$, or children suspected for abuse related to a head injury ( 23.0 versus $9.1 \%, p<0.001)$ were more likely to have fractures detected with skeletal survey.

A few recent studies have reported the utility of followup skeletal survey in the determination of child abuse [25, 26]. Follow-up skeletal survey can be utilized to detect fractures that in their acute stage are undetectable on skeletal survey but appear some time after injury on radiology due to callous formation in the bone healing process. One study looked retrospectively at a consecutive sample of children who received a follow-up skeletal survey in the course of 7 years at one children's hospital [26]. Fourteen percent of the group, receiving both baseline and follow-up skeletal survey, had additional fractures identified in the follow-up films. More importantly, 8 of these children had definitive abuse determination based on the result of their follow-up skeletal survey. Additionally, a secondary analysis of multi-site data, investigated the results of follow-up skeletal survey in children evaluated for physical abuse by 20 different child abuse investigation teams in the United States [25]. Over $15 \%$ of the sample had fractures identified on repeat skeletal survey, with half having multiple fractures identified. Seven percent of the population with positive follow-up skeletal survey had no findings on original skeletal survey. An equal number of children were deemed not to have fractures that were originally suspected in baseline skeletal survey. For $34 \%$ of patients, the clinical teams' suspicion for abuse increased after followup skeletal survey results were available.

\section{Use of ALT/AST in Determining Abdominal Trauma}

Intra-abdominal injuries are uncommon although deadly in child abuse victims. Because intra-abdominal injuries are unlikely to occur in common "alibi" mechanisms for child abuse such as stairway falls [27-29], their identification in suspected child abuse can often help to elucidate mechanism. Lindberg et al. [30] performed a retrospective data analysis to examine the appropriate threshold for transaminase testing for occult abdominal injury. The study found that a cut-off of $80 \mathrm{IU} / \mathrm{L}$ was an optimal level for detection of abdominal trauma, with a $77 \%$ sensitivity and $82 \%$ specificity. Higher cut-offs which have been previously recommended including 100 and $400 \mathrm{IU} / \mathrm{L}$ showed less optimal results with sensitivities of 68 and $40 \%$, respectively. A more recent study by this same group validated these findings [31]. They examined over 1,500 patients suspected of child abuse whose transaminases were measured. The predetermined cut-off of $>80 \mathrm{IU} / \mathrm{L}$ was associated with a $83 \%$ sensitivity and $83 \%$ specificity for predicting abdominal injury. In this study, lipase and amylase did not prove to be optimal for the detection of occult abdominal injury.

\section{Use of CT in Determining Head Trauma}

Head trauma is the leading cause of death in child abuse and these injuries tend to occur in the very young. What is of concern for the clinician is that these infants with iTBI (inflicted traumatic brain injury) can present with nonspecific symptoms such as vomiting, fussiness, and ALTE. They often do not present with neurological symptoms, making the diagnosis of iTBI difficult $[32,33]$. Because of this, CT is often recommended in these children. With the known radiation-associated cancer risk of CT [34], we should be looking at alternatives to narrow the population 
that are required to undergo CT in order to identify a child abuse mechanism.

\section{Biomarkers in Inflicted Head Trauma (iTBI)}

The misdiagnosis of iTBI is common, as the diagnosis of iTBI can be very difficult. Caregivers rarely provide information on the history of trauma and children present with non-specific symptoms such as ALTEs, fussiness, and the physical exam can be normal [35-40]. Multiple studies [41-44] have shown that children with iTBI also tend to have a higher mortality, more severe injuries and probably have worse outcomes when compared to non-inflicted TBI (nTBI). Confounding the picture of inflicted TBI is the possibility of a history of earlier maltreatment or unreported previous TBI [45]. Literature has pointed to the connection between diffuse hypoxic-ischemic brain injury patterns and their association with abusive head trauma [46]. Because of this, increase in the use of MRI is being studied for its utility in abusive head trauma.

There is currently no specific well-established screening test to help physicians identify children with iTBI who present with non-specific symptoms and might benefit from a head CT. It would be very helpful if we could predict which children had iTBI and if we could predict their outcome. Prediction of outcome in younger children is very difficult. Standards such as glascow coma scale (GCS) and glascow outcome score (GOS) used in adult patients for the rating of impaired consciousness may have limited utility in children, as it relies on both motor and verbal abilities often not yet developed in young children [47, 48].

The use of serum biomarkers as indicators of severity of brain injury and therefore outcome is very compelling in predicting how these patients will recover and maybe help with identifying the mechanism that caused the TBI. The hypothesis is that these biomarkers are released from the brain with injury, then pass into CSF fluid, then into the blood, and can then be measured in the serum to identify the need for radiological testing and/or the presence of a TBI.

Biomarkers that have been studied include serum NSE (Neuron specific enolase), S100B (S100 calcium Binding Protein B), and MBP (myelin basic protein) [49, 50]. The literature suggests that the higher the biomarker levels the worse the patient's outcome. Berger et al. [35] studied whether serum and/or cerebrospinal fluid concentrations of NSE, S100B, and MBP are sensitive and specific for iTBI in high risk infants. A prospective study was performed on 98 infants with no history of trauma that presented with non-specific symptoms such as ALTE, vomiting without diarrhea or fever, seizure or seizure-like activity, or nonspecific neurological findings of lethargy or fussiness. All patients enrolled were later classified as having the presence or absence of TBI. NSE was $76 \%$ sensitive and $66 \%$ specific for identifying head injury in this population. MBP was only $36 \%$ sensitive, but $100 \%$ specific for head injury. S100B was elevated in the vast majority of all patients in the study and thus was not reasonable to use to detect head injury. Serum and CSF concentrations of NSE and MBP have the potential to be used as screening tests for TBI in well-appearing infants who present with nonspecific symptoms [35].

Understanding the effect of iTBI and outcome after TBI is very important. Infants with iTBI may have a history of earlier maltreatment or unreported TBI, and this can also confuse the predicted outcome after injury. Beers et al. [51] studied the effect of iTBI on neurocognitive outcome in children less than 12 years of age, assessing the relationship between serum biomarker measured at the time of injury and outcome. They evaluated neuro serum biomarkers (initial levels, time to peak levels and peak levels for each biomarker) and 6 month cognitive and functional outcome in 30 infants and children who had presented with inflicted TBI $(n=15)$ or non-inflicted TBI $(n=15)$ before the age of 3 years. The serum biomarkers studied included NSE, S100B, and MBP. The outcome measures were evaluated at 6 months post TBI and included assessing personal and social sufficiency of patients (Vineland Adaptive Behavior Scale), intellectual development (Bayley Scales of Infant Development), and intellectual ability (Stanford-Binet Intelligence Scale-IV). They found that children with iTBI were younger and more likely to sustain a subdural hematoma and less likely to sustain a skull fracture. The inflicted TBI group had worse GOS scores and poorer adaptive abilities and lower intellectual development at follow-up. For all three serum biomarkers, time to peak levels were also higher in the iTBI group. This study would suggest that children with iTBI are at increased risk for a poorer outcome and therefore requires more extensive rehabilitation [51-53]. A possible explanation is that children with iTBI have brain injuries that are delayed in diagnosis and treatment. The longer time to peak of biomarkers in iTBI compared to nTBI was hypothesized to be secondary to a delay in seeking medical care, delay in correct diagnosis, and may involve various levels of hypoxemia.

\section{Conclusions}

Child abuse is a public health epidemic in the United States with high incidence, prevalence, and severe personal and societal impact. Surgeons should be considering child abuse in their treatment of children with traumatic injury. Common risk factors identified during hospital stay associated with child abuse determination include caregiver 
behavior and social risks, inconsistent history, trauma history not consistent with developmental ability, trauma history inconsistent with injuries, specific fracture types, bruising and fractures in the non-ambulatory, and delayed presentation. Screening for child abuse mechanism in the ED and in in-patient settings is not precise and is documented to be biased and incomplete. Many tools and factors have been studied for their predictive potential though studies have a number of methodological issues and no one screening tool has been proposed as standard of care. More research is warranted to better define how to screen patients for the need for further child abuse investigation. Research shows that both initial and follow-up skeletal surveys are an important modality for screening and detection of child abuse-related fractures and are instrumental in identifying mechanisms for many children under investigation. Abdominal trauma is an important differential in child abuse determination as they are unlikely to be caused by common low-impact traumatic events. Because of this, we should consider transaminase levels in the evaluation of suspected child abuse. The cut-off for need for further evaluation including $\mathrm{CT}$ of the abdomen is transaminase levels of $>80 \mathrm{IU} / \mathrm{L}$ which showed optimum sensitivity and specificity in one study. Additional research would strengthen this recommendation. Finally, the use of head $\mathrm{CT}$ in children under suspicion for inflicted head trauma is necessary as many patients present asymptomatic. Due to the known cancer risk associated with $\mathrm{CT}$, this modality should be limited, though very few additional screening modalities exist to limit the number of children needing a head CT. Studies on serum CSF biomarkers of head injury are promising and further research is needed for the recommendation of consistent use in the clinical setting.

\section{Compliance with Ethics Guidelines}

Conflict of Interest Nilda M. Garcia and Karla A. Lawson declare that they have no conflict of interest.

Human and Animal Rights and Informed Consent This article does not contain any studies with human or animal subjects performed by any of the authors.

\section{References}

Papers of particular interest, published recently, have been highlighted as:

- Of importance

1. W.H. Organization. Definition of child maltreatment. http://www. WHO.int/topics/child_abuse/en/. 2014.

2. US Department of Health and Human Services Administration for Children and Families. Child Maltreatment. 2012.
3. Felitti VJ, Anda RF, Nordenberg D, Williamson DF, Spitz AM, Edwards V, Koss MP, Marks JS. Relationship of childhood abuse and household dysfunction to many of the leading causes of death in adults. The adverse childhood experiences (ACE) study. Am J Prev Med. 1998;14:245-58.

4. Helfer M, Kempe RS, Krugman RD. The battered child. Chicago: The University of Chicago Press; 1997.

5. - Larimer EL, Fallon SC, Westfall J, Frost M, Wesson DE, NaikMathuria BJ. The importance of surgeon involvement in the evaluation of non-accidental trauma patients, J Pediatr Surg. 2013;48:1357-1362. This article presents interesting findings regarding the need for surgeon involvement in the determination of child abuse based on severity and occult nature of injuries and need for surgical intervention.

6. Deans KJ, Thackeray J, Askegard-Giesmann JR, Earley E, Groner JI, Minneci PC. Mortality increases with recurrent episodes of nonaccidental trauma in children. J Trauma Acute Care Surg. 2013;75:161-5.

7. Jenny C, Hymel KP, Ritzen A, Reinert SE, Hay TC. Analysis of missed cases of abusive head trauma. JAMA. 1999;281:621-6.

8. Ravichandiran N, Schuh S, Bejuk M, Al-Harthy N, Shouldice M, $\mathrm{Au} \mathrm{H}$, Boutis K. Delayed identification of pediatric abuse-related fractures. Pediatrics. 2010;125:60-6.

9. Lane WG, Rubin DM, Monteith R, Christian CW. Racial differences in the evaluation of pediatric fractures for physical abuse. JAMA. 2002;288:1603-9.

10. Bailhache M, Leroy V, Pillet P, Salmi LR. Is early detection of abused children possible? A systematic review of the diagnostic accuracy of the identification of abused children. BMC Pediatr. 2013;13:202.

11. Chang DC, Knight VM, Ziegfeld S, Haider A, Paidas C. The multi-institutional validation of the new screening index for physical child abuse. J Pediatr Surg. 2005;40:114-9.

12. Hettler J, Greenes DS. Can the initial history predict whether a child with a head injury has been abused? Pediatrics. 2003;111:602-7.

13. Pierce MC, Kaczor K, Aldridge S, O'Flynn J, Lorenz DJ. Bruising characteristics discriminating physical child abuse from accidental trauma. Pediatrics. 2010;125:67-74.

14. Valvano TJ, Binns HJ, Flaherty EG, Leonhardt DE. Does bruising help determine which fractures are caused by abuse? Child Maltreat. 2009;14:376-81.

15. Vinchon M, de Foort-Dhellemmes S, Desurmont M, Delestret I. Confessed abuse versus witnessed accidents in infants: comparison of clinical, radiological, and ophthalmological data in corroborated cases. Childs Nerv Syst. 2010;26:637-45.

16. Vinchon M, Defoort-Dhellemmes S, Desurmont M, Dhellemmes P. Accidental and nonaccidental head injuries in infants: a prospective study. J Neurosurg. 2005;102:380-4.

17. Wells RG, Vetter C, Laud P. Intracranial hemorrhage in children younger than 3 years: prediction of intent. Arch Pediatr Adolesc Med. 2002;156:252-7.

18. • Louwers EC, Korfage IJ, Affourtit MJ, Ruige M, van den Elzen AP, de Koning HJ, Moll HA. Accuracy of a screening instrument to identify potential child abuse in emergency departments. Child Abuse Negl. 2013. This review investigates a number of different screening modalities for child abuse detection and presents these findings in an aggregate format.

19. Hymel KP, Willson DF, Boos SC, Pullin DA, Homa K, Lorenz DJ, Herman BE, Graf JM, Isaac R, Armijo-Garcia V, Narang SK, Pediatric Brain Injury Research Network Investigators. Derivation of a clinical prediction rule for pediatric abusive head trauma. Pediatr Crit Care Med. 2013;14:210-20.

20. Berger R, McGinn T. Deciding whether to screen for abusive head trauma: do we need a clinical decision rule? Pediatr Crit Care Med. 2013;14:230-1.

21. Higginbotham N, Lawson KA, Gettig K, Roth J, Hopper E, Higginbotham E, George TM, Maxson T, Edwards G, Garcia NM. 
Utility of a child abuse screening guideline in an urban pediatric emergency department. J Trauma Acute Care Surg. 2014;76(3): $871-7$.

22. Kemp AM, Dunstan F, Harrison S, Morris S, Mann M, Rolfe K, Datta S, Thomas DP, Sibert JR, Maguire S. Patterns of skeletal fractures in child abuse: systematic review. BMJ. 2008;337:a1518.

23. Section on Radiology, American Academy of Pediatrics. Diagnostic imaging of child abuse. Pediatrics. 2009;123:1430-5.

24. - Duffy SO, Squires J, Fromkin JB, Berger RP. Use of skeletal surveys to evaluate for physical abuse: analysis of 703 consecutive skeletal surveys. Pediatrics. 2011;127:e47-52. This work highlights the utility of skeletal surveys in the diagnosis of child abuse.

25. Harper NS, Eddleman S, Lindberg DM, Ex SI. The utility of followup skeletal surveys in child abuse. Pediatrics. 2013;131:e672-8.

26. Singh R, Squires J, Fromkin JB, Berger RP. Assessing the use of follow-up skeletal surveys in children with suspected physical abuse. J Trauma Acute Care Surg. 2012;73:972-6.

27. Chiaviello CT, Christoph RA, Bond GR. Stairway-related injuries in children. Pediatrics. 1994;94:679-81.

28. Huntimer CM, Muret-Wagstaff S, Leland NL. Can falls on stairs result in small intestine perforations? Pediatrics. 2000;106:301-5.

29. Rivara FP, Alexander B, Johnston B, Soderberg R. Populationbased study of fall injuries in children and adolescents resulting in hospitalization or death. Pediatrics. 1993;92:61-3.

30. Lindberg D, Makoroff K, Harper N, Laskey A, Bechtel K, Deye $\mathrm{K}$, Shapiro R, Investigators U. Utility of hepatic transaminases to recognize abuse in children. Pediatrics. 2009;124:509-16.

31. Lindberg DM, Shapiro RA, Blood EA, Steiner RD, Berger RP, ExSTRA Investigators. Utility of hepatic transaminases in children with concern for abuse. Pediatrics. 2013;131:268-75.

32. Greenes DS, Schutzman SA. Clinical indicators of intracranial injury in head-injured infants. Pediatrics. 1999;104:861-7.

33. Laskey AL, Holsti M, Runyan DK, Socolar RR. Occult head trauma in young suspected victims of physical abuse. J Pediatr. 2004;144:719-22.

34. Brenner DJ, Hall EJ. Computed tomography: an increasing source of radiation exposure. N Engl J Med. 2007;357:2277-84.

35. Berger RP, Dulani T, Adelson PD, Leventhal JM, Richichi R, Kochanek PM. Identification of inflicted traumatic brain injury in well-appearing infants using serum and cerebrospinal markers: a possible screening tool. Pediatrics. 2006;117:325-32.

36. Duhaime AC, Partington MD. Overview and clinical presentation of inflicted head injury in infants. Neurosurg Clin $\mathrm{N}$ Am. 2002;13:149-54.

37. Ettaro L, Berger RP, Songer T. Abusive head trauma in young children: characteristics and medical charges in a hospitalized population. Child Abuse Negl. 2004;28:1099-111.

38. Haviland J, Russell RI. Outcome after severe non-accidental head injury. Arch Dis Child. 1997;77:504-7.

39. Morris MW, Smith S, Cressman J, Ancheta J. Evaluation of infants with subdural hematoma who lack external evidence of abuse. Pediatrics. 2000;105:549-53.
40. O'Neill JA Jr, Meacham WF, Griffin JP, Sawyers JL. Patterns of injury in the battered child syndrome. J Trauma. 1973;13:332-9.

41. Barlow K, Thompson E, Johnson D, Minns RA. The neurological outcome of non-accidental head injury. Pediatr Rehabil. 2004;7:195-203.

42. Irazuzta JE, McJunkin JE, Danadian K, Arnold F, Zhang J. Outcome and cost of child abuse. Child Abuse Negl. 1997;21:751-7.

43. Keenan HT, Runyan DK, Marshall SW, Nocera MA, Merten DF. A population-based comparison of clinical and outcome characteristics of young children with serious inflicted and noninflicted traumatic brain injury. Pediatrics. 2004;114:633-9.

44. Perez-Arjona E, Dujovny M, DelProposto Z, Vinas F, Park H, Lizarraga S, Park T, Diaz FG. Late outcome following central nervous system injury in child abuse. Childs Nerv Syst. 2003;19:69-81.

45. Beers SR, De Bellis MD. Outcomes of child abuse. Neurosurg Clin N Am. 2002;13:235-41.

46. Kadom N, Khademian Z, Vezina G, Shalaby-Rana E, Rice A, Hinds T. Usefulness of MRI detection of cervical spine and brain injuries in the evaluation of abusive head trauma. Pediatric Radiol. 2014;44:839-48.

47. Durham SR, Clancy RR, Leuthardt E, Sun P, Kamerling S, Dominguez T, Duhaime AC. CHOP Infant Coma Scale ("infant face scale"): a novel coma scale for children less than two years of age. J Neurotrauma. 2000;17:729-37.

48. Teasdale GM, Pettigrew LE, Wilson JT, Murray G, Jennett B. Analyzing outcome of treatment of severe head injury: a review and update on advancing the use of the Glasgow outcome scale. J Neurotrauma. 1998;15:587-97.

49. Berger RP, Adelson PD, Pierce MC, Dulani T, Cassidy LD, Kochanek PM. Serum neuron-specific enolase, S100B, and myelin basic protein concentrations after inflicted and noninflicted traumatic brain injury in children. J Neurosurg. 2005;103:61-8.

50. Savola O, Hillbom M. Early predictors of post-concussion symptoms in patients with mild head injury. Eur $\mathrm{J}$ Neurol. 2003;10:175-81.

51. Beers SR, Berger RP, Adelson PD. Neurocognitive outcome and serum biomarkers in inflicted versus non-inflicted traumatic brain injury in young children. J Neurotrauma. 2007;24:97-105.

52. Barlow KM, Thomson E, Johnson D, Minns RA. Late neurologic and cognitive sequelae of inflicted traumatic brain injury in infancy. Pediatrics. 2005;116:e174-85.

53. Ewing-Cobbs L, Kramer L, Prasad M, Canales DN, Louis PT, Fletcher JM, Vollero H, Landry SH, Cheung K. Neuroimaging, physical, and developmental findings after inflicted and noninflicted traumatic brain injury in young children. Pediatrics. 1998;102:300-7. 